\title{
La derecha en la crisis del Bicentenario
}

\author{
Fabián Bustamante Olguín ${ }^{1}$
}

\section{Hugo Herrera. Santiago de Chile: Ediciones Universidad Diego Portales, 2014, 213 páginas.}

Los estudios sobre la derecha chilena cuentan con un vasto recorrido durante el siglo XX sobre todo en lo que refiere a sus ideas políticas y a su papel en la justificación del golpe de Estado de 1973 y la dictadura militar. Sin embargo, el libro que se reseña plantea un importante desafío para el estudio de la derecha en el Chile post-dictadura: repensar su falta de comprensión política. Al respecto, cabe señalar que si bien es cierto que algunos de sus representantes políticos poseen tribuna en los medios de comunicación, existe poco esfuerzo intelectual en sus intervenciones públicas lo que impide su inserción en el debate político actual. Todo ello, por cierto, puede verificarse en los innumerables adjetivos calificativos para referirse a los enemigos políticos.

En este contexto, el filósofo y director del Instituto de Humanidades de la Universidad Diego Portales, Hugo Herrera, nos presenta este libro precedido de varios artículos publicados en el diario La Tercera en calidad de columnista. Desde ya el título advierte una crisis en la derecha, una crisis intelectual producto de la falta de un discurso renovado, generando superficialidad a la hora del debate político en una sociedad chilena mucho más compleja y dinámica, ${ }^{2}$ a pesar de su primer gobierno en democracia con Sebastián Piñera (2010-2014). De ahí que el autor sostenga la nula articulación de una respuesta (o propuesta) a futuro. Las consecuencias de esta derecha desajustada (históricamente, a mi juicio) de la experiencia diaria de la realidad nacional y desterrar su diversa historia intelectual es lo que pretende ilustrarnos el autor.

El libro está conformado por cinco capítulos, sumado un anexo con comentarios de los siete últimos libros que se han publicado en los últimos años sobre la derecha.

Ya en el prefacio del libro, el autor sostiene que la derecha chilena todavía conserva un discurso propio de la Guerra Fría que combina una concepción

Chileno. Magíster en Historia, mención Chile, Universidad de Santiago de Chile. Académico, Escuela de Sociología de la Universidad Católica Silva Henríquez y Facultad de Ciencias Físicas y Matemáticas, Universidad de Chile. E-mail: fgbustamanteo@gmail.com

2 Una crisis acentuada aún más por el "caso PENTA" y el financiamiento ilegal para las campañas políticas principalmente al partido de derecha Unión Demócrata Independiente (UDI). Entre los beneficiados por PENTA (holding empresarial chileno con inversiones en distintas áreas como seguros, finanzas, salud, del cual es socio fundador el ex yerno de Augusto Pinochet, Carlos Alberto Délano) están los senadores UDI, Jovino Novoa, Ena Von Baer e Iván Moreira, sumado al precandidato presidencial de ese partido, Lawrence Golborne. 
individualista del derecho de propiedad, la idea de subsidiariedad y de democracia protegida. Este discurso es difícil de sostener en los tiempos actuales cuando el enemigo comunista claramente ya no existe. Esto ha provocado una insuficiencia argumentativa para enfrentarse a discusiones más complejas y, lo que es peor aún, una baja en el apoyo popular del sector.

En este marco el autor pone énfasis en el capítulo primero titulado Cambio de Ciclo, una interesante demostración del cambio de ciclo que vive nuestro país, enfatizando en las alteraciones que dan cuenta del desequilibro existente entre pueblo e institucionalidad. Tales alteraciones -que afectan tanto a la derecha como a la izquierda- serían: la disminución del miedo, debilitamiento de los ejes del pasado reciente, distribución del conocimiento y la información, oligopolio, oligarquía, centralismo, empobrecimiento espiritual, la revolución (C'est une révolution) y el romanticismo político. Estas variaciones no alcanzarían a ser una revolución para cambiar el modelo y sólo se manifestarían en forma de revuelta.

En el capítulo segundo, titulado Comprensión Política, se expone un criterio de lo que Herrera entiende por una comprensión específicamente política, que requiere superar la reducción mecánica de las situaciones concretas para acercarse contemplativamente hacia la realidad. Lo que propone el autor es que la derecha inicie una apertura a la realidad y utilice un marco teórico lo suficientemente complejo para analizar el escenario actual.

En relación con lo anterior, por su parte, en el capítulo tres, titulado Una mirada a la historia intelectual de la derecha en Chile, el autor propone rescatar las distintas tradiciones intelectuales que en su momento articularon un pensamiento de derecha, capaz de debatir con sólidos argumentos. Por cierto, este pensamiento estaría conformado por dos vertientes: la nacionalpopular y la socialcristiana, agregándose la síntesis entre conservantismo y liberalismo económico. Dentro de ella se destacan a Francisco Encina, Alberto Edwards (nacional-popular), Mario Góngora (socialcristiano) y Jaime Guzmán (conservantismo y liberalismo económico). Al respecto, este capítulo del libro constituye el punto central de la tesis de Herrera, a saber: en la derecha existió un aparato conceptual más sofisticado durante el siglo XX que le permitió pensar y actuar tal la realidad política de ese momento (como lo hizo Encina con la crisis del Centenario). A partir de este análisis, Herrera considera que la derecha debería reencontrarse con estas vertientes de pensamiento caracterizadas por la unión entre pensamiento y acción política. Unión que, por cierto, no existe en la actualidad, provocando una pérdida en el terreno de las "estructuras de poder legítimo" (universidades, sindicatos, etc.).

En el cuarto capítulo, El desafío comprensivo de la derecha chilena, Herrera sugiere una particular alternativa a la crisis de la derecha acudiendo a una 
actitud de contemplación (un no hacer nada o suspensión del activismo), permitiendo así un conocimiento más cabal de las cuatro tradiciones discursivas de la derecha ordenadas bajo dos ejes: una liberal/no liberal y la cristiana/laica, de la que luego vienen las combinaciones. Por lo que la tradición cristiana-liberal se expresaría en la UDI y en parte de Renovación Nacional; la socialcristiana en el antiguo Partido Conservador, Falange Nacional y en algunos movimientos contemporáneos como Solidaridad de la Universidad Católica; la liberal-laica con raíces en el Partido Liberal, en Amplitud y de forma parcial en Renovación Nacional; y finalmente la laica/nacional-popular que comienza con el ibañismo extendiéndose en el Partido Agrario-Laborista, Partido de Acción Nacional y Partido Nacional para esparcirse en diversas iniciativas como Avanzada Nacional y Frente Nacional del Trabajo, dejando huella en las raíces fundacionales de Renovación Nacional. A partir de esta clasificación, el autor propone reactivarlas (entendiéndolo como una aproximación reflexiva a las fuentes de las tradiciones, atenuando los extremos de todas ellas) con el propósito de encontrar allí ideas o argumentaciones que permiten la elaboración de un discurso diverso con capacidad de reconocimiento para el ciudadano común. Reconocimiento que, por cierto, logra la Nueva Mayoría (hoy en el gobierno).

En el último capítulo, Síntesis y aspectos fundamentales de una esperable nueva conciencia en la derecha chilena, realiza una síntesis de los otros capítulos, subrayando en que el momento actual le exige a la derecha un discurso más denso y sofisticado, incluyendo teoría y filosofía política, y no sólo discusiones de carácter técnico. Para ello debe volver a su historia intelectual y abrirse paso a la realidad nacional. Todo ello, en efecto, permitirá -afirma el autor- alcanzar legitimidad política.

En el anexo incorpora el autor algunos libros de representantes de la derecha. Los títulos de estos libros son: La fatal ignorancia. La anorexia cultural de la derecha frente al avance ideológico progresista (2009), de Axel Káiser; Gobernar con principios: Ideas para una nueva derecha (2012), de Francisco Javier Urbina y Pablo Ortúzar; El malestar de Chile ¿Teoría o diagnóstico? (2012), de Marcel Oppliger y Eugenio Guzmán; Chile camino al desarrollo. Avanzando en tiempos difíciles (2012), del ex ministro de Piñera Cristián Larroulet; El regreso del modelo (2012), de Luis Larraín; Con la fuerza de la libertad, La batalla por las ideas de centro-derecha en el Chile de Hoy (2013), de Jovino Novoa; y Virar derecha. Historia y desafíos de la centro-derecha en Chile (2014), del ex diputado UDI Gonzalo Arenas. No obstante, es importante señalar que algunos de ellos como Cristián Larroulet y Jovino Novoa intentan realizar un esfuerzo intelectivo de elaborar observaciones acerca de la realidad presente, pero no dan cuenta de la crisis de discurso o algún camino de solución para remediarlo. 
Pese a los planteamientos de Herrera sobre la crisis de la derecha chilena, el trabajo presenta algunas limitaciones.

Lo primero que cabe decir al respecto es que la principal dificultad que presenta esta obra es sobre cuál derecha se está refiriendo el autor. Quizás sea más apropiado -siguiendo el título de Sandra McGee- hablar de las "derechas". ${ }^{3}$ En ese sentido quisiera señalar que la mayor parte del discurso político y filosófico de la "derecha" proviene de la extrema derecha, y no de la derecha partidaria. Siguiendo la idea de Octavio Rodríguez Araujo, la extrema derecha (o ultraderecha) es más ideológica que la derecha (en este caso la UDI, RN y los sectores económicos), que es mucho más pragmática, pues su ideología es de base empírica-positivista, sobre todo cuando tiene el poder (que por definición es pragmático). ${ }^{4}$ En ese sentido, la derecha actual -que es heredera y defensora del modelo económico impuesto por Pinochet-, no está muy interesada en la ideología (o en los debates intelectuales) sino en conservar su statu quo. Por lo que "la ideología de la derecha -siguiendo a Rodríguez Araujo- es la defensa de sus intereses y los que representa, tenga o no el poder gubernamental". Por lo tanto, una derecha que tiene el poder de facto de nuestro país -a pesar de los sucesivos gobiernos de la Concertación-, difícilmente estará interesada en buscar una tradición intelectual de derecha -mencionada en el libro-, tradición intelectual que debe encontrarse -a mi juicio- en la extrema derecha.

Por cierto que Francisco Encina, Alberto Edwards y Jaime Guzmán -señalados por Herrera- estarían dentro de esa línea. Precisamente estos autores no son mencionados como parte de la derecha antidemocrática y antiliberal. Cuestión que constituye una dificultad para el propósito del autor de que la derecha actual deba rescatar estos discursos ideológicos no democráticos. Cabe destacar que Alberto Edwards, con su libro Bosquejo histórico de los partidos políticos chilenos, de 1903, inauguraría una tradición de pensamiento antidemocrático que culminaría con el dictador Augusto Pinochet. Todos ellos justifican un régimen dictatorial del cual exhortan a terminar con el desorden propio del sistema demoliberal y devolverle a Chile el orden que estaría encarnado en la figura de Portales. ${ }^{5}$ Estos rasgos distintivos de jerarquía y autoridad formaron parte de la identidad del pensamiento conservador chileno durante el siglo XX, -y a pesar de sus diferencias y conflictividad internas entre algunas tendencias dentro de ella (nacionalistas y tradicionalistas)-, tuvieron un elemento en co-

3 Ver Sandra McGee (2005), Las derechas: la extrema derecha en la Argentina, el Brasil y Chile, 1890-1939, Buenos Aires: Universidad Nacional de Quilmes.

$4 \quad$ Véase al respecto, Octavio Rodríguez Araujo (2004), Derechas y ultraderechas en el mundo. México: Ediciones Siglo XXI.

5 Ver Luis Corvalán Márquez (2009), Nacionalismo y autoritarismo durante el siglo en Chile. Santiago: Editorial Universidad Católica Silva Henríquez. 
mún: su visceral anticomunismo, en especial durante el período de la Unidad Popular, que morigera tales conflictividades y pluralidades. ${ }^{6}$

De otro lado, como se señaló más arriba, si el autor pretende hablar de tradiciones intelectuales, le faltó mencionar la revista Estudios, que posee una gran densidad conceptual (a partir de la década del treinta), depositada en autores como Jaime Eyzaguirre, Julio Phillipi, Roque Esteban Scarpa, entre otros, comprometidos todos ellos con el tradicionalismo hispánico. ${ }^{7}$ Aquí hay un primer indicio de que la derecha estuvo inserta dentro del ámbito de las humanidades, aunque construyendo un marco conceptual que sirvió para dar forma a discursos legitimantes de la destrucción de la democracia y la instauración de la dictadura militar.

Cabe, finalmente, hacer notar que, en todo caso, estos intelectuales de derecha (Alberto Edwards, Francisco Encina o el mismo Jaime Guzmán) recepcionaron ideas provenientes del pensamiento conservador antiliberal europeo de finales del siglo XVIII e inicios del siglo XIX, que reaccionó contra la Revolución Francesa, corriente que Carl Schmitt denominó como "pensamiento contrarrevolucionario", y que luego "nacionalizaron" de acuerdo a las realidades propias de nuestro país. ${ }^{8}$ Tal recepción, como lo señala Luis Corvalán Márquez, estaría dada por las vertientes tradicionalistas y nacionalistas. Lo cual no permite sostener la idea de un pensamiento nacional. Al respecto considero que al indagar en una tradición intelectual de derecha habría que prestar atención a la extrema derecha. Demás está decir que, al mismo tiempo, ésta sólo recepciona las ideas antiliberales y antidemocráticas europeas, restándole originalidad al pensamiento de derecha.

Digamos, por último, que la falta de discurso y de densidad intelectiva en la derecha implicaría cuestionar el sistema neoliberal heredado de la dictadura de Pinochet, del cual ellos son herederos y defensores. Quizás sea ésta la principal razón de la carencia discursiva que Herrera argumenta en su obra, y hasta ahora, no se aprecia un cambio significativo a futuro. En ese sentido -y como tal como lo expone el autor en el anexo- sus personeros no están dispuestos ni a autoanalizarse como sector político como tampoco proponer un discurso que no sea lo que habitualmente nos tiene acostumbrados la derecha: poco análisis, obcecada y llena de simplismos.

6 Al respecto, véase el artículo de Ernesto Bohoslasvky "¿Qué es lo nuevo de la nueva derecha en Chile? Anticomunismo, corporativismo y neoliberalismo, 1964-1973", en História Unisinos, 16: 5-14.

7 Véase el interesante estudio de Isabel Jara (2007) De Franco a Pinochet. El proyecto cultural franquista en Chile 1936-1980. Santiago: Editorial Universidad de Chile.

8 Por ejemplo, el caso del hacendado-ensayista Francisco Encina "nacionalizó" el pensamiento racista de Osvaldo Spengler. Ilevando a cabo su interpretación de la Historia de Chile. 